\title{
Autoimmune Encephalitis as an Extra-articular Manifestation of Rheumatoid Arthritis
}

\author{
Eiji Kitamura, Yuko Kondo, Naomi Kanazawa, Tsugio Akutsu, \\ Kazutoshi Nishiyama and Takahiro Iizuka
}

\begin{abstract}
:
Autoimmune encephalitis (AE) is an immune-mediated encephalitis characterized by the subacute onset of memory deficits, altered mental status, or psychiatric symptoms. Limbic encephalitis associated with rheumatoid arthritis (RA) has not been reported yet. A 57-year-old man presented with the subacute onset of headache, depression, and anorexia 7 months before the onset of RA. Brain magnetic resonance imaging showed symmetric parenchymal lesions involving the medial temporal lobes. He was diagnosed with RA and AE, but no autoantibodies to neuronal intracellular or cell-surface antigens were identified in either the serum or cerebrospinal fluid. His symptoms improved with immunotherapy. AE can develop as an extra-articular manifestation of RA.
\end{abstract}

Key words: autoimmune encephalitis, rheumatoid arthritis, rheumatoid pachymeningitis, autoantibodies

(Intern Med 58: 1007-1009, 2019)

(DOI: 10.2169/internalmedicine.1846-18)

\section{Introduction}

Rheumatoid arthritis (RA) is an immune-mediated inflammatory disease characterized by persistent joint inflammation with a varied course ranging from spontaneously remitting synovitis to aggressive ankylosing, severely disabling joint disease (1). RA is also known to be a systemic disease often associated with cutaneous and organ-specific extraarticular manifestations (1). Although the central nervous system (CNS) is rarely involved in RA, a variety of neurological manifestations occur associated with vasculitis, pachymeningitis, meningitis, opportunistic infection, drug adverse effects, or other comorbid rheumatologic disorders (2-4). A case of meningoencephalitis with leptomeningeal enhancement was previously reported (5), but autoimmune encephalitis (AE) with extensive changes in the parenchymal brain magnetic resonance imaging (MRI) findings associated with RA has not been reported yet.

We herein report a case of $\mathrm{AE}$ that developed at the preclinical stage of RA.

\section{Case Report}

A 57-year-old previously healthy white man began to have frontal throbbing headaches 7 months before admission. He subsequently became depressive and lost his appetite. Five months before admission, he noticed hand tremors, dizziness, and weight loss of $13 \mathrm{~kg}$ for 18 months. He was referred and admitted to our hospital. His medical history was unremarkable. He had a family history of RA in his elder brother.

On admission, the temperature was $36.2^{\circ} \mathrm{C}$. The findings on a physical examination were unremarkable, and he had no arthralgia or joint swelling. On a neurologic examination, he was alert and well-oriented but depressive. The minimental state examination score was 28 . He had mild shortterm memory loss, but his cognitive function was otherwise normal. He had postural hand tremors, rigidity in the upper extremities, hyperreflexia, and truncal ataxia. The neck was supple. The laboratory tests showed elevated levels of serum CRP $(1.47 \mathrm{mg} / \mathrm{dL})$, ESR $(59 \mathrm{~mm} / \mathrm{h})$, ANA (1:320 dilution), rheumatoid factor $(\mathrm{RF})(313 \mathrm{IU} / \mathrm{mL}$, normal $<15)$, cyclic citrullinated peptide (CCP) antibodies $(1,570 \mathrm{U} / \mathrm{mL}$, normal 

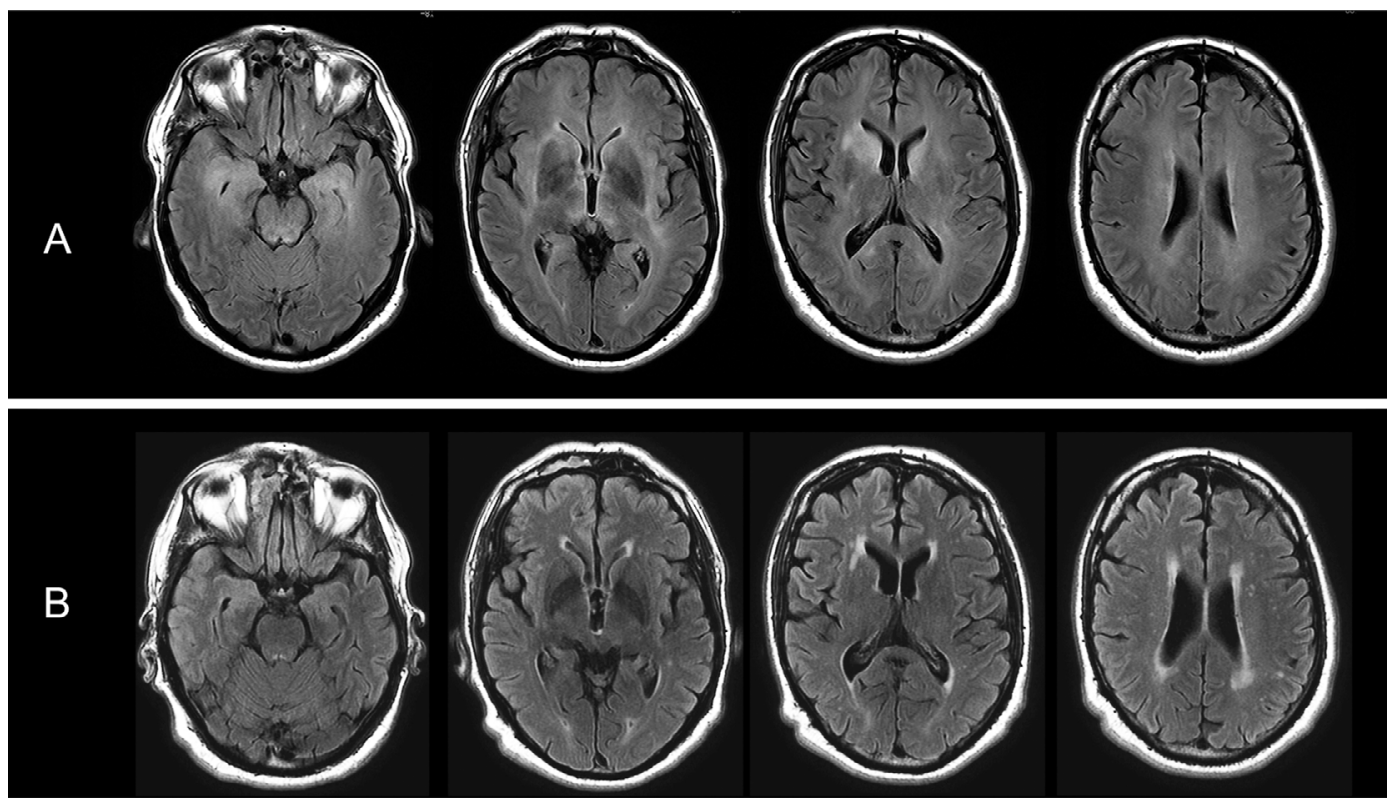

Figure. Brain MRI findings. Brain MRI obtained on admission shows symmetrical increased fluidattenuated inversion recovery signals in the medial temporal lobes, caudate head, and periventricular white matter, with minimum gadolinium enhancement (A). Follow-up brain MRI obtained 20 months later shows marked improvement in the increased FLAIR signals (B).

$<4.5)$, sIL-2R (834 U/mL, normal <519), and Tg-antibodies (40 IU/mL, normal $<28$ ), but the thyroid function was normal. Serum MMP-3, ProGRP, and antibodies to DNA, Sm, SS-A/Ro, SS-B/La, TPO, and MPO-ANCA findings were all negative. A cerebrospinal fluid (CSF) examination revealed 18 white blood cells (WBCs)/ $\mu \mathrm{L}$ (mononuclear cells 94\%), an elevated protein level $(101 \mathrm{mg} / \mathrm{dL})$, and a normal glucose level $(63 \mathrm{mg} / \mathrm{dL})$. The IgG index $(3.52$, normal $\leq 0.73)$, IL-6 (18.9 pg/mL), and CCP antibodies $(217 \mathrm{U} / \mathrm{mL})$ were elevated, but RF was not elevated (4 IU/mL) in the CSF. Three oligoclonal bands (OCBs) were detected. Polymerase chain reaction testing for herpes simplex virus 1 and 2 was negative. The findings of a cytological examination were normal. Autoantibodies to classical paraneoplastic anti-neuronal antigens [CV2/CRMP5, Ma2/Ta, Ri, Yo, Hu, Recoverin, SOX1, Titin, Zic4, GAD65, Tr (DNER), and amphiphysin] were examined in serum with EUROLINE (Euroimmun AG) and determined to be negative. Autoantibodies to neuronal cellsurface antigens [NMDA receptor, AMPA receptor, GABA (A) receptor, GABA(B) receptor, mGluR5, Caspr2, DPPX, and LGI1] were measured at the laboratory of Josep Dalmau (University of Barcelona) using both immunohistochemistry on rat brain tissue and cell-based assays, but no autoantibodies were detected in either the serum or CSF. Rat brain tissue immunohistochemistry did not reveal any immunostaining for neuropils. HLA genotype showed A1, A3, B8, and B 35 , but B51 and B53 were not seen; Cw1 was not examined. Brain MRI showed symmetrical MRI abnormalities in the medial temporal lobes, caudate head, and periventricular white matter (Figure A), but no apparent leptomeningeal or parenchymal enhancement was seen. An electroencephalogram (EEG) showed slowing without paroxysmal discharge.
Whole-body computed tomography (CT) showed no evidence of a tumor.

At admission (day 1), the patient was initially suspected of having limbic encephalitis or primary CNS lymphoma. However, on day 2, he began to complain of joint pain with swelling in his shoulder, elbow, and proximal and distal interphalangeal joints of the 3rd and 4th fingers, suggesting RA. The laboratory results supported the diagnosis of RA. He began to receive intravenous high-dose methylprednisolone (IVMP; 1,000 mg, 5 days) from day 12, followed by oral prednisolone $(50 \mathrm{mg} / \mathrm{day})$, of which the dosage was gradually tapered. On day 17 , rigidity, tremor, and truncal ataxia started to improve. On day 23 , his headache, depression, arthralgia, and swelling were also resolved. On day 44, oral methotrexate was started in order to treat RA. On day 48 , he was discharged without residual neurological symptoms.

Follow-up brain MRI obtained 20 months later showed marked improvement (Figure B). He remained free of symptoms on prednisolone $(5 \mathrm{mg} /$ day $)$ and methotrexate $(8 \mathrm{mg}$ once weekly) at 24 months after symptoms presentation without a clinical relapse of symptoms.

\section{Discussion}

To our knowledge, this is the first case of AE with extensive parenchymal lesions that developed at the preclinical stage of RA as the first extra-articular manifestation of RA, without other comorbid systemic disorders or the presence of autoantibodies against either neuronal intracellular antigens or neuronal cell-surface antigens. The diagnostic criteria for possible, probable, and definite $\mathrm{AE}$ were proposed in 
2016 (6), with rheumatologic disorders, such as RA or lupus, listed in the differential diagnosis of AE. However, the present case illustrated that AE can develop associated with RA.

A histopathological examination of the brain was not performed. Accordingly, the histopathological findings remain unclear; however, the subsequent recovery of the neurological symptoms, resolution of MRI abnormalities following immunotherapy, and the presence of OCBs with an elevated IgG index strongly support an autoimmune etiology. The absence of serological markers suggesting vasculitis, skin lesions, peripheral neuropathy, ischemic brain lesions, or perivascular enhancement does not support a vasculitic etiology of the brain lesions. Primary CNS lymphoma is also unlikely. Therefore, AE can be recognized as an extra-articular manifestation of RA, along with rheumatoid meningitis or pachymeningitis $(3,7)$.

Rheumatoid pachymeningitis is known to develop at any stage of RA or even many years after the onset of RA (3); however, it can also precede the onset of RA (8). Rheumatoid pachymeningitis or meningitis develops regardless of the disease severity of RA or its duration (7), but there have been no reports of a case in which AE involving the medial temporal lobes developed as an extra-articular manifestation of RA.

The CSF levels of RF have been used as a diagnostic marker of rheumatoid meningitis (7). The CSF levels of IL$6(7)$ or CCP-antibodies $(5,9)$ are also reported to be correlated with the clinical symptoms of rheumatoid meningitis. In this case, RF was not elevated in the CSF, but both IL-6 and CCP-antibodies were elevated in the CSF, although the exact upper limit of the normal CSF levels is not available. These data may support an autoimmune etiology associated with RA.

Although no treatment strategy has been established yet for either rheumatoid meningitis or pachymeningitis, anecdotal case series (7) demonstrated a high mortality rate of up to $60 \%$ in cases treated with corticosteroids alone. Therefore, we started IVMP and methotrexate, resulting in the resolution of the symptoms. The patient remains free of symptoms on prednisolone and methotrexate.

In conclusion, $\mathrm{AE}$ can develop at the preclinical stage of RA without the presence of autoantibodies to neuronal intracellular or cell-surface antigens and responds to aggressive immunotherapy.

This study was approved by Institutional Review Boards of Kitasato University (B17-144).
Author's disclosure of potential Conflicts of Interest (COI).

Kazutoshi Nishiyama: Research funding, Nippon Boehringer Ingelheim, Daiichi Sankyo, Astellas Pharma, Otsuka Pharmaceutical, Kyorin Pharmaceutical, Dainippon Sumitomo Pharma, Teijin Pharma, Nihon Pharmaceutical, Pfize, Bristol-Myers Squibb, Japan Blood Products Organization, MSD and Nihon MediPhysics.

Financial Support: This work was supported in part by a grant from Japan Epilepsy Research Foundation (JERFTENKAN 17002).

\section{Acknowledgement}

We particularly thank Professor Josep Dalmau in Service of Neurology, IDIBAPS Hospital Clínic, University of Barcelona, Casanova (Spain) for examining autoantibodies against neuronal cell-surface and synaptic proteins.

Eiji Kitamura and Takahiro Iizuka contributed equally to this work.

\section{References}

1. Prete M, Racanelli V, Digiglio L, Vacca A, Dammacco F, Perosa F. Extra-articular manifestations of rheumatoid arthritis: An update. Autoimmun Rev 11: 123-131, 2011.

2. Rodríguez Sanz A, Tallón Barranco A, Díez-Tejedor E. Autoimmune-induced focal meningoencephalitis. Neurologia 28: 63-64, 2013.

3. Starosta MA, Brandwein SR. Clinical manifestations and treatment of rheumatoid pachymeningitis. Neurology 68: 1079-1080, 2007.

4. Palazzo E, Yahia SA. Progressive multifocal leukoencephalopathy in autoimmune diseases. Joint Bone Spine 79: 351-355, 2012.

5. Shibahara T, Matsushita T, Matsuo R, et al. Anti-cyclic citrullinated peptide antibody-positive meningoencephalitis in the preclinical period of rheumatoid arthritis. Case Rep Neurol 8: 156160, 2016.

6. Graus F, Titulaer MJ, Balu R, et al. A clinical approach to diagnosis of autoimmune encephalitis. Lancet Neurol 15: 391-404, 2016.

7. Kato T, Hoshi K, Sekijima Y, et al. Rheumatoid meningitis: an autopsy report and review of the literature. Clin Rheumatol 22: 475-480, 2003.

8. Padjen I, Mayer M, Habek M, Kolenc D, Dotlić S. Redefining a diagnosis: from meningeal plasma cell granuloma to rheumatoid meningitis. Report of a patient follow-up. Neurol Sci 36: 10471048, 2015.

9. Kim HY, Park JH, Oh HE, Han HJ, Shin DI, Kim MH. A case of rheumatoid meningitis: pathologic and magnetic resonance imaging findings. Neurol Sci 32: 1191-1194, 2011.

The Internal Medicine is an Open Access journal distributed under the Creative Commons Attribution-NonCommercial-NoDerivatives 4.0 International License. To view the details of this license, please visit (https://creativecommons.org/licenses/ by-nc-nd/4.0/).

(C) 2019 The Japanese Society of Internal Medicine Intern Med 58: 1007-1009, 2019 